\title{
Performance Evaluation of the IEEE 802.15.4 MAC for Low-Rate Low-Power Wireless Networks
}

\author{
Gang Lu, Bhaskar Krishnamachari, Cauligi S. Raghavendra \\ Department of Electrical Engineering, University of Southern California \\ Los Angeles, CA 90089 \\ \{ganglu, bkrishna, raghu\}@usc.edu
}

\begin{abstract}
IEEE 802.15.4 is a new standard to address the need for low-rate low-power low-cost wireless networking. We provide in this paper one of the first simulation-based performance evaluations of the new medium access protocol in IEEE 802.15.4, focusing on its beacon-enabled mode for a star-topology network. We describe its key features such as the superframe structure, which allows devices to access channels in a contention access period (CAP) or a collision free period (CFP) and the beacon-based synchronization mechanism. Our performance evaluation study reveals some of the key throughput-energy-delay tradeoffs inherent in this $M A C$ protocol. We provide an analysis comparing the energy costs of beacon tracking and nontracking modes for synchronization, showing that the optimum choice depends upon the combination of duty cycles and data rates.
\end{abstract}

\section{Introduction}

In the next few years, it is expected that low rate wireless personal area networks (LR-WPAN) will be used in a wide variety of embedded applications, including home automation, industrial sensing and control, environmental monitoring and sensing. In these applications, numerous embedded devices running on batteries are distributed in an area communicating via wireless radios. Compared to wireless local area networks (WLAN) which aim to provide highthroughput, low-latency for traditional file transfer and multimedia applications, the required data rate for LR-WPAN applications is expected to be only on the order of tens of kbps. Similarly, the required message latency may be on the order of $100 \mathrm{mis}$ or more [4]. However the key concern in these applications is that of extremely low power consumption, since it is often infeasible or undesirable to replace or recharge batteries for the devices on a regular basis.

The MAC protocol plays a significant role in determining the efficiency of wireless channel bandwidth sharing and energy cost of communication. IEEE 802.11 [2] for wireless local network (WLAN) is an "overkill technology" for LR-WPAN applications because IEEE 802.11 and its variants consume significant energy in order to provide high data rate which is not required for LR-WPANs. Another relevant technology is Bluetooth (IEEE 802.15.1) [3], which also allows moderately high data rates (allowing, for example, audio traffic), small networks ( 8 nodes per PAN) and does not focus primarily on energy-efficiency as it is intended for devices that are either main powered or regularly rechargeable.

Recently, a new standard named IEEE 802.15.4 has been developed [1], [5]. The goal of IEEE 802.15.4 is to provide a physical-layer and MAC-layer standard with ultra-low complexity, cost, and power for lowdata-rate wireless connectivity among cheap fixed devices.

The IEEE 802.15.4 standard supports multiple network topologies, including both star and peer-to-peer networks depending on the application requirements. In this paper, we will evaluate the MAC performance of IEEE 802.15.4 in the simple star-topology. In the star topology, the communication is established between a single central controller, called the PAN coordinator and up to 255 other devices. Such startopologies may also exist inside clusters in a larger network of 802.15.4 devices.

We now present in section 2 an overview of the IEEE 802.15.4 MAC protocol. We will then present our analysis and simulation results in section 3 .

\section{Overview of the MAC protocol in IEEE 802.15.4}

The MAC protocol in IEEE 802.15.4 can operate on both beacon enabled and non-beacon modes. In the beaconless mode, the protocol is essentially a simple CSMA-CA protocol. Since most of the unique features of IEEE 802.15.4 are in the beacon-enabled mode, we will focus our attention on this mode. 


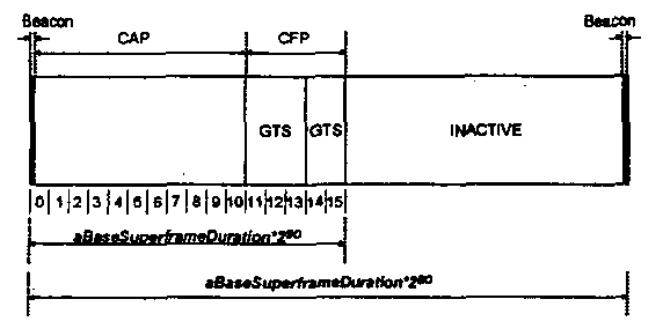

Figure 1: An illustration of the IEEE 802.15.4 superframe structure (from [1]).

In beacon mode, the IEEE 802.15.4 uses a superframe structure. Figure 1 illustrates this structure. A superframe begins with beacon frames sent periodically by the coordinator at an interval that can ranges from $15 \mathrm{~ms}$ to $245 \mathrm{~s}$. There are both active and inactive portion in the superframe. Devices communicate with their PAN only during the active period and enter a low power mode during the inactive period. The parameter macBeaconOrder $(B O)$ decides the length of beacon interval $(B I=$ $2^{B O} \times a B a s e$ SuperFrameDuration) and the parameter macSuperframeOrder $(S O)$ describes the length $\left(2^{S O} \times\right.$ aBaseSuperFrameDuration $)$ of the active portion of the superframe. The active portion of each superframe is further divided into 16 equal time slots and consists of three parts: the beacon, a Contention Access Period (CAP) and a Collision Free Period (CFP) (which is only present if guaranteed time slots (GTS) slots are allocated by the PAN coordinator to some of the devices). Each GTS consists of some integer multiple of CFP slots and up to 7 GTS are allowed in CFP. We now present some key features of IEEE 802.15.4 MAC.

\subsection{Data Transfer}

A node wishing to send data to the PAN coordinator needs to receive a beacon to understand the current superframe structure. If it has been allocated a GTS, it sends its data during the CFP, otherwise, it sends its data using CSMA-CA in the CAP.

For communications from the coordinator to the devices, in order to allow devices to be in power saving mode at their own will to save energy, transaction requests can be initiated from the devices themselves rather than from the coordinator. A device sends a data request commands to the coordinator during the CAP if its address is in the data pending list of the beacon. The coordinator sends an acknowledgement frame with a flag indicating that data is forthcoming and sends the packet afterwards. Once the data is re- ceived, the device sends an acknowledgement back to the PAN coordinator.

\subsection{Low Duty cycle}

The coordinator announces the superframe structure to devices in the PAN periodically through beacon frames. By changing the active and inactive portion via the parameters $S O$ and $B O$, the WPAN can operate under low duty cycle to conserve energy.

\subsection{Contention Access Period (CAP)}

The CAP starts right after the beacon and before the CFP on a superframe, and all frames in the CAP use slotted CSMA-CA as follows.

Three variables are maintained at each device for a channel access: $N B, C W$ and $B E . N B$ is the number of times the CSMA-CA backoffs while attempting the current transmission, and is rest to 0 for each new data transmission. $C W$ is the contention window length, which is reset to 2 either for a new data transmission or when the channel is found to be busy. $B E$ is the backoff exponent, which is related to the backoff periods a device should wait before attempting carrier sensing.

When a device need to transmit during the CAP, it enables its receiver and delays for a random number of complete backoff periods (up to $2^{B E}-1$ periods) and then determines if the channel is clear. The MAC ensures that, after the random backoff, the remaining CSMA-CA operations can be undertaken and the entire transaction can be completed before the end of CAP. If the channel is busy, the MAC increases both $N B$ and $B E$ by one, and resets $C W$ to 2 . If $N B$ is less than or equal to macMaxCSMABackof $f s$, the CSMA-CA delays for a random time again, otherwise it terminates with a failure.

In CSMA-CA, a lot of energy is generally consumed by the long backoff period which is required during high traffic periods to avoid collision. However, IEEE 802.15.4 supports a Battery Life Extension (BLE) mode, in which the CSMA-CA backoff exponent is limited to the range 0-2. This reduces the period of idle listening in low offered traffic applicar tions. A network device can put its radio to sleep to conserve energy immediately after the reception of acknowledgement packet if there is no more data to be sent or received.

\subsection{Collision Free Period (CFP)}

The IEEE 802.15.4 standard allows the optional use of CFP for devices that require dedicated bandwidth to achieve low latencies. The.CFP is between the slot boundary immediately following the CAP and the start of the next beacon. All contention-based transactions must be completed before the CFP begins. 
Table 1: Radio parameters

\begin{tabular}{|c|c|}
\hline Radio bandwidth & $250 \mathrm{Kbps}$ \\
\hline Radio Transmission Range & $16 \mathrm{~m}$ \\
\hline Transmit Power & $31 \mathrm{~mW}$ \\
\hline Receive Power & $35 \mathrm{~mW}$ \\
\hline Idle Power & $30 \mathrm{~mW}$ \\
\hline
\end{tabular}

A device requiring dedicated bandwidth or lowlatency transmission can be assigned a guaranteed time slot (GTS) in CFP by the PAN coordinator. When a device wishes to transmit a frame using GTS, it first checks a list on the beacon frame to see whether it has been allocated a valid GTS. If a valid GTS is found, the device enables its receiver at a time prior to the start of the GTS and transmits the data during the GTS period. The MAC of the PAN coordinator ensures that its receiver is enabled for all allocated guaranteed time slots.

\subsection{Synchronization}

The PAN coordinator transmits beacon frames periodically to announce the superframe structure in a PAN. Devices need to synchronize with a coordinator by receiving and decoding the beacon frames before any data transmission. There are two methods of synchronization: tracking and non-tracking.

With tracking, the device receives the first beacon, gets current superframe structure, knows when to active its receiver for the next beacon and keep track of it. To transmit a frame, the device can enable its receiver just a little earlier before the beacon arrival. With non-tracking, the device attempts to acquire the beacon only once. The device needs to enable its receiver and searches for a specific period until it receives a beacon from its associated coordinator, when it attempts to transmit a frame.

\section{Performance Evaluation}

We now describe our performance evaluation study of the IEEE 802.15.4 MAC. We implemented our prototype in the NS-2 network simulator with the CMU wireless extension. The radio characteristics are shown in Table 1 (from [7]). The network topology used in the simulation is a $7 \times 7$ grid (i.e. 49 nodes) with $4 m$ distance between adjacent nodes. The node at the center is the PAN coordinator. All other nodes are in the radio transmission range of the coordinator, but not all nodes are in a single broadcast domain. All simulations are run independently and their results averaged under 10 different seeds. Unless otherwise specified, sources generate packets at constant averaged rate with $50 \%$ randomization in inter-packet

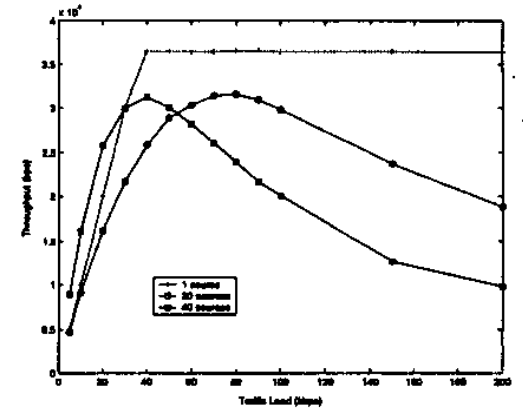

Figure 2: Throughput of CSMA-CA in beaconenabled IEEE 802.15.4.

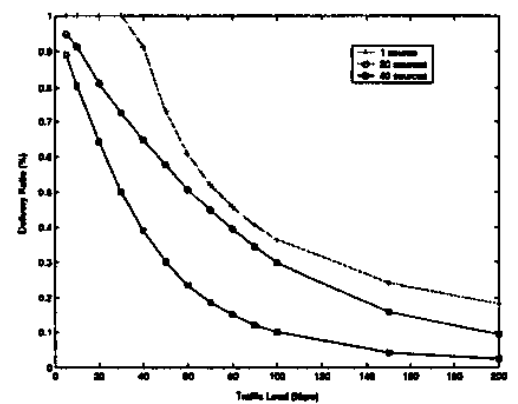

Figure 3: Delivery Ratio of CSMA-CA in beaconenabled IEEE 802.15.4.

interval. The performance metrics we evaluated are: energy, latency, throughput and delivery ratio.

\subsection{CSMA-CA in CAP}

The CSMA-CA algorithm in IEEE 802.15.4 employs a small backoff period, i.e. Battery Life Extension (BLE), to reduce the idle listening energy consumption. This is based on the assumption of low traffic applications in WPAN. The beacon boundary in the superframe structure requests a device not to transmit a frame if the remaining time in the CAP is not enough to complete the transaction, which could cause low channel utilization efficiency.

Figures 2 and 3 show the throughput and delivery ratio for different numbers of sources sending 50 byte packets with an exponential inter-arrival time. In these simulations, we choose $S O=0$ and $B O=0$, which makes the the duty cycle to be $100 \%$. The active portion is $0.01536 \mathrm{~s}$, same as the beacon interval, and the beacon frame is 24 bytes. All sources access the channel via CSMA-CA during CAP.

We find that the highest throughput is achieved, when there is only 1 source, at around $38 \mathrm{kbps}$. Note that this is substantially below the nominal value of 


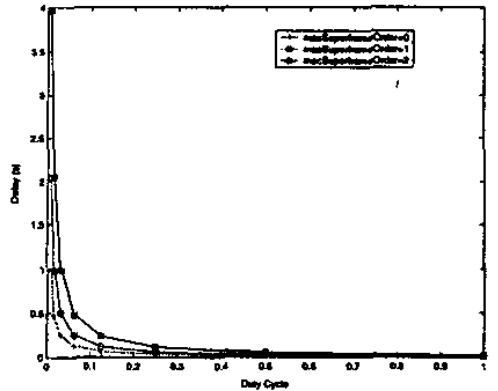

Figure 4: Delay as a function of duty cycle.

$250 \mathrm{kbps}$ due to the presence of random backoffs, ACK messages, as well as a large setting for inter-frame spacing; higher bandwidths are possible with other settings, particularly in CFP mode. When there are muitiple sources, the throughput increases when the total offered traffic load is less than 70kbps then begins to decrease because of the collision. The highest throughput with multiple sources is seen to be about $32 \mathrm{kbps}$.

\subsection{Duty Cycle}

In IEEE 802.15.4, the coordinator decides the duty cycle and beacon interval that the PAN operates on. This is done by setting the $S O$ and $B O$ parameters explained earlier. The duty cycle is given by the expression $\frac{2^{s O}}{2^{B O}}$. Low duty cycle conserves energy by putting devices to sleep. However, a low duty cycle reduces the bandwidth and increases latency. With the same duty cycle, we can have different combination of $S O$ and $B O$ which means the superframe structure has different active period and inactive period.

In this simulation, one device generates packets at $100 \mathrm{bps}$ to the coordinator for 2000 seconds. Figure 4 and 5 show that as the duty cycle decreases, energy consumption decreases linearly (Beacon reception consumes most of the energy while data transmission energy cost is negligible.) However, the packet delivery delay increases exponentially as the duty cycle decreases.

With same duty cycle, the smaller $S O$ which means smaller active period and beacon interval also consumes more energy. This is because in a fixed simulation time, the smaller beacon interval means more beacon frames transmitted/received. However, the latency is smaller as a packet which arrives at the inactive portion needs to wait a shorter period.

\subsection{Guaranteed Time Slots}

As noted before, guananteed time slots can be assigned in the CFP to applications which require dedi-

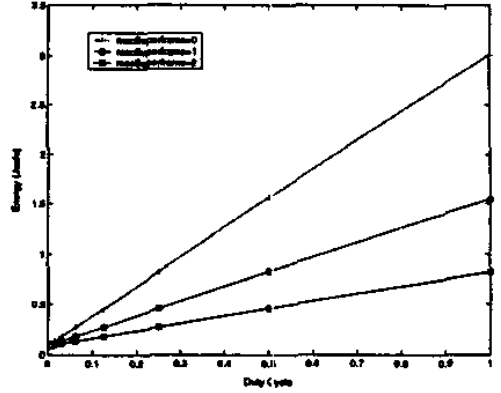

Figure 5: Energy consumption of source device as a function of duty cycle.

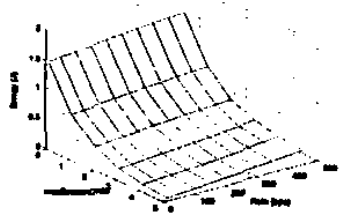

(a) tracking

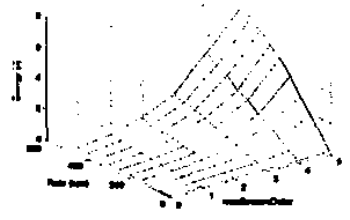

(b) non-tracking
Figure 7: Energy cost of a device with tracking as a function of duty cycle and data rate.

cated bandwidth or low latency regardless of the traffic load in the network. However, as in TDMA, the time slot assigned to an application could be wasted if there is no data transmission request. In addition, a device with GTS has to track the beacon frames from the coordinator periodically in order to transmit data during the GTS slot assigned to it.

In this simulation, we compare the delay, energy and throughput performance for a source using CSMA-CA and GTS under various background traffic. There are 20 sources as the background traffic which transmit CBR data during CAP. Figure 6(a), 6 (b) and 6(c) show the results. Clearly, with GTS, a device can get dedicated bandwidth which achieves $100 \%$ delivery ratio and low latency regardless of the background traffic load in the network. However, the energy consumption increases as the result of beacon frame receptions.

\subsection{Synchronization}

Before a device can transmit any data frame, it needs to synchronize with the coordinator by receiving a beacon frame. With beacon tracking, a device enables its radio periodically to receive beacon frame 


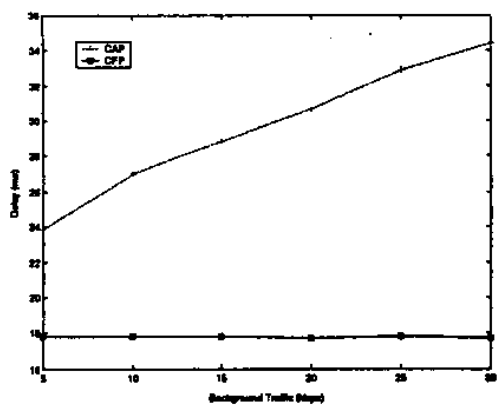

(a) latency

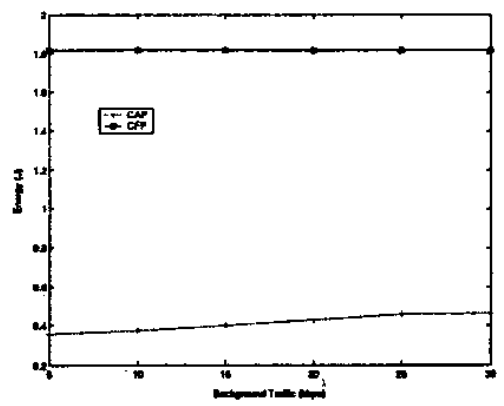

(b) energy

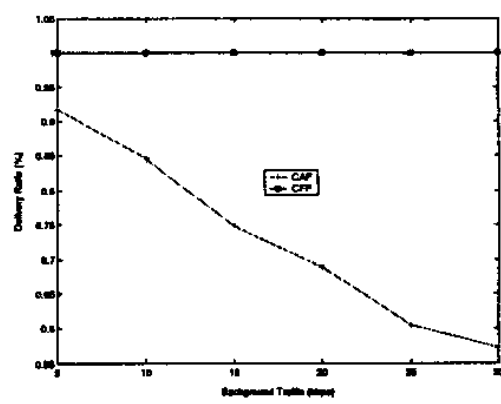

(c) ratio

Figure 6: Relative performance of CAP and CFP modes for a source under different background traffic.

which consumes energy. However, because the device knows the time for next beacon frame, it can keep sleeping until that time, which may conserve energy. On the contrary, without beacon tracking, a device does not spend énergy on periodical beacon reception. However, since it does not know where the beacon appears, it has to enable its radio immediately to search a beacon when it need to communicate with the coordinator, which may incur high energy cost due to long idle period.

There are tradeoffs between tracking and nontracking depending on the duty cycle and data rate. When duty cycle is low, for a fixed period, the number of beacon frames is reduced. So the beacon tracking consumes less energy to receive the beacon frames. Meanwhile non-tracking devices need to wait longer time statistically before they can receive a beacon frame, which increases energy consumption. With higher data rate, the probability of a data packet needing to be transmitted in a beacon interval is increased. At the same time there is also a need to acquire the synchronization for non-tracking device, and thus the energy consumption would also increase.

In the simulation, we compared the energy cost of a device with tracking and non-tracking. The active portion of the superframe is fixed at $S O=0$. The duty cycle is decreased by increasing beacon interval with larger $B O$. The traffic is from a single device with CBR data. All data rate is lower than the bandwidth capacity.

Figure 7(a) and 7(b) show the energy cost of tracking and non-tracking. The energy costs of both tracking and non-tracking increase as data rate increases. However, when duty cycle increase, with tracking the energy cost increases while without tracking the en- ergy cost decreases.

\subsubsection{Energy Cost Analysis of Tracking versus Non-Tracking Modes}

We perform a simple analysis to evaluate the tradeoff. Let $P_{t}, P_{r}, P_{i}$ be the power of radio transmission, reception and idle listening; let $T_{b}, T_{d}, T_{a}, T_{i}$ be the time duration of beacon frame, data frame, acknowledgement frame and random backoff respectively; let $p$ be the probability that there is a packet to be sent in a beacon interval $B I$. Suppose the data rate is $r$ and packet size is size bytes, $p=r \cdot B I /$ size $\cdot 8$. For the low rate WPAN, we assume there is at most one data frame in a beacon interval.

With Tracking, the energy cost in a superframe beacon interval consists of beacon frame receptions and if there is a frame transmission, the energy consumption of the backoff period, the frame transmission and the acknowledgement frame reception:

$$
E_{\text {Tracking }}=P_{r} T_{b}+p\left(P_{t} T_{d}+P_{r} T_{a}+P_{i} T_{i}\right)
$$

Without tracking, the energy cost in a superframe beacon interval is zero if there is no frame need to be sent. If there is a packet need to be sent, the device need to listen for $B I / 2$ on average in order to synchronize with the coordinator then send the packet in the next beacon interval.

$$
E_{N o n-T r a c k i n g}=p\left(P_{i} \frac{B I}{2}+P_{t} T_{d}+P_{r} T_{a}+P_{i} T_{i}\right)
$$

Figure 8 shows a comparison of the energy costs of tracking and non-tracking for a $25 \%$ duty cycle scenario. We find that the analysis results match with the 


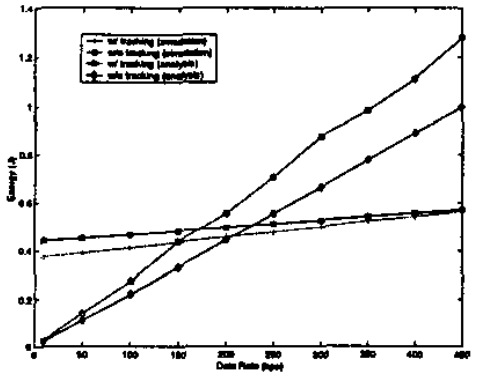

Figure 8: Comparison of energy cost of source with tracking and non-tracking.

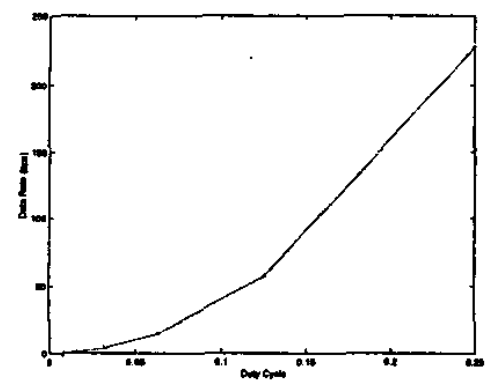

Figure 9: Crossover curve for the tracking/nontracking tradeoff.

simulation result quite well. Figure 9 plots the crossover curve, i.e. the data-rate and duty-cycle combinations for which the two modes have the same energy cost. For data rates and duty-cycles above this curve, tracking mode is preferable to non-tracking, and vice versa.

\section{Conclusion and Future Work}

We have described the new IEEE 802.15.4 MAC standard for low-rate low-power wireless networks, with a focus on the beacon-enabled MAC for startopology networks. Based on NS-2 simulations, we evaluate the performance of various features in the IEEE 802.15.4 MAC. We find that extremely low duty cycle operation enables significant energy saving, but that these savings can come at the cost of significantly higher latency and lower bandwidth. The CSMA-CA algorithm reduces energy cost due to idle listening in the backoff period but increases the collision at higher rate and larger number of sources. While the use of GTS in the contention-free period can allow dedicated bandwidth to a device to ensure low latency, the device need to track the beacon frames in this mode, which increases the energy cost. We also analyzed the tradeoff between tracking or non-tracking modes for synchronization, and showed that this depends upon the duty cycle and data rate.

One direction for future work would be to employ real experiments to test the performance of IEEE 802.15.4 when products become available. Another is to evaluate the performance of this protocol in peerto-peer topologies. In the meantime, to benefit the research community, we are working to make our NS2 implementation of this protocol publicly available online.

\section{References}

[1] 802.15.4-2003 IEEE Standard for Information Technology-Part 15.4: Wireless Medium Access Control (MAC) and Physical Layer (PHY) specifications for Low Rate Wireless Personal Area Networks (LR-WPANS), 2003.

[2] 802.11-1999 IEEE Stana'ard for Information Technology - LAN/MAN - Specific requirements - Part 11: Wireless LAN Medium Access Control (MAC) and Physical Layer (PHY) specifications, 1999.

[3] 802.15.1-2002 IEEE Standard for information technology - Telecommunication and information exchange between systems - LAN/MAN - Part 15.1: Wireless Medium Access Control (MAC) and Physical Layer (PHY) specifications for Wireless Personal Area Networks(WPANs), 2002.

[4] Ed Callaway, Paul Gorday and Lance Hester, "Home Networking with IEEE 802.15.4: A Developing Standard for Low-Rate Wireless Personal Area Networks", in IEEE Communications Magazine, August 2002

[5] J. A. Gutierrez et al., "IEEE 802.15.4: A Developing standard for Low-Power, Low-Cost Wireless Personal Area Networks", in IEEE Networks, Sept. 2001

[6] Jose A. Gutierrez, Edgar H. Callaway, Jr., and Raymond L. Barrett, Jr., "Low-Rate Wireless Personal Area Networks: Enabling Wireless Sensors with IEEE 802.15.4", IEEE Press, 2003

[7] Data Sheet for CC2420 2.4GHz IEEE 802.15.4/Zigbee RF Transceiver, available online at http://www.chipcon.com/files/ CC2420_Data_Sheet_1_0.pdf 